\title{
Simulation of simultaneous photonic and phononic band gaps in Sapphire
}

\author{
H. Bentarki ${ }^{1}$,, M. Tlidi ${ }^{2}$, and A. Makhoute ${ }^{1}$ \\ ${ }^{1}$ Physique du Rayonnement et des Interactions Laser-Matière, Faculté des Sciences. Moulay Ismail University. B.P. 11201, \\ Zitoune, Meknès, Morocco. \\ ${ }^{2}$ Faculté des Sciences, Université Libre de Bruxelles (U.L.B.), CP. 231, Campus Plaine, B-1050 Bruxelles, Belgium.
}

\begin{abstract}
The simultaneous existence of photonic and phononic band gaps in a simple square periodic array of holes drilled in sapphire substrate is investigated theoretically. The investigations are focused in a first part on a photonic gap obtaining with a phononic gap in the same time making use of some specific parameters. In the second part, using a cavity defect in which the strong confinement of acoustic and optic waves enhances the interaction, we obtain photonic and phononic modes classified according to their symmetry (even or odd), also allowed bands in gaps, which has led to the propagation of waves appropriate. The frequencies of the localized modes in the first band gap are computed with a finite element method.
\end{abstract}

\section{Introduction}

The photonic band gap materials [1-2] on one hand and phononic [3-4] on the other hand generate a significant interest in the scientific community in recent years. They are structures having a periodicity of one or more spatial directions. This periodic variation leads to the appearance of photonic and phononic bands which are defined as the frequency ranges for which any kind of wave cannot propagate in the structure, whatever its polarization and direction of propagation. These properties make these interesting crystals for many applications in integrated optics for the realization of new components such micro cavities with high quality factor, micro guides with low losses, micro sources lasers and spatial filters in wavelength (super prisms, filters,...). A new path is open in the field of acousto-optic devices [5-6 and 7] through the simultaneous existence of photonic and phononic structures, seeking to consolidate the principle of photonic and phononic crystal in one structure named phoXonic crystal. The interest of such artificial crystal is simultaneously confining of optical and acoustic energy. Such a structure is interesting for the study of interactions between photons and phonons but also for the development and improvement of devices of integrated acousto-optic, such modulators or multiplexers. The theme of $2 \mathrm{D}$ phoxonic crystals is very recent since the first theoretical papers published are from 2006 [8-9], when Maldovan and Thomas [10-11] showed theoretically that the phononic and photonic bandgap can be obtained in $2 \mathrm{D}$ crystals for a square or hexagonal network of air holes in a silicon matrix. Thereafter, the method of supercells [12] demonstrated the existence of localized modes, for confining optical and an acoustic wave in a cavity. Sadat-Saleh et al. [13] demonstrated the possibility of opening the phononic and photonic band gaps in complex matrices such as multiple cylinders (more than one atom per unit cell) into 2D structures in Lithium Niobate. The studies of periodic structures of pillars which al-

a e-mail: bentarki .houda@gmail .com low the existence of forbidden bands were conducted, various structures containing only air inclusions [14-15]. The acousto-optical interaction started since the 60's, thanks to the invention of the laser and Leon Brillouin in 1914, which made the first theory of the coupling of a light wave with a hyper-sonic wave [16]. Leon Brillouin theory, published in 1921, provides that a liquid traversed by a hypersonic wave causes periodic variations of constraints that create, by photo-elastic effect, changes in the refractive index, then behaving as a network of diffraction for light. [16] This has resulted in the following years, a series of studies both theoretical and experimental involving photonphonon interaction. Other studies, this time based on other than a 2D square lattice structures, [17-18] have emerged. For example using plates, [19] or the use of structured nano wave guides [20-21 and 22].

The main goal of this paper is to conduct a theoretical study of simultaneous phononic and photonic band gaps, in 2D square lattice constituted by a periodic array of holes deposited on a layer. We concentrate our calculations on a structure where the holes and the supporting plate are respectively made of air and sapphire. Taking sapphire as a matrix component allows us to take advantage of the anisotropy of the dielectric tensor in the microwave regime to show the possibility of confining optical energy in both polarizations (TE and TM) and energy acoustic simultaneously. In general, the phononic and photonic band structures are calculated by finite element (FE). However, finite difference time domain (FDTD) method has also been used in 2010 for the same structure, [23] just that we chose a different lattice parameter, to have localized modes at the introduction of defects in the perfect structure.

The paper is structured as follows. In section 2, we describe the geometrie considered in this paper as well as the method of calculation. This section presents also analysis of obtained results, and section 3 concludes the paper. 


\section{Numerical method, results and discussion}

\subsection{Geometry and methodology}

From the first work on photonic and phononic crystals, and after the publication of Yablonivitch in 1987, [24] the complexity of experimental studies and their cost have emerged. Then, the need for development of optical modeling methods for the study of these structures is imposed. It is then necessary to solve Maxwell's equations [25] for the study of photonic crystals and the elasticity equations for phononic crystals. To solve these equations numerically, some methods have been developed, such as the finite element [FE] method, as we have chosen to achieve our goal. The structure we used for this study was elaborated in a sapphire plate with a square lattice of cylindrical air holes drilled through the plate (see Fig. 1(a)). The lattice constant ( $a=$ $70(\mathrm{~nm})$ ) was set to this value in order to obtain a photonic and phononic band gap as large as possible in this structure. For the same reason, the filling ratio was fixed to about its maximum value $(f=0.66)$, leaving only a few nanometers between two adjacent holes. The unit-cell used for computation of the dispersion relation and the modes of a phoxonic crystal slab is shown in Fig. 1(b). By replacing one hole in the middle of the structure, we created a substitution-type defect forming a cavity in the phoxonic crystal, Fig. 1 (c) shows this structure.

The table 1 summarizes the acoustic and optical parameters of the material used in simulations.

Table 1. Please write your table caption here.

Table 2. Elastic and optical constants of Sapphire $\left(\mathrm{Al}_{2} \mathrm{O}_{3}\right)$ cubic considered and used in numerical simulations. The refractive index $(\mathrm{n})$, the density $(\rho)$, the longitudinal $\left(c_{l}\right)$ and transverse $\left(c_{t}\right)$ velocities of sound along the [100] direction of propagation [2627].

\begin{tabular}{|l|l|l|l|l|l|}
\cline { 2 - 6 } \multicolumn{1}{c|}{} & $\mathrm{n}$ & $\rho\left(\mathrm{Kg} / \mathrm{m}^{3}\right)$ & $\varepsilon$ & $c_{t}(\mathrm{~m} / \mathrm{s})$ & $c_{l}(\mathrm{~m} / \mathrm{s})$ \\
\hline Sapphire & 1.77 & 3950 & 11.5 & 6040 & 11090 \\
\hline
\end{tabular}

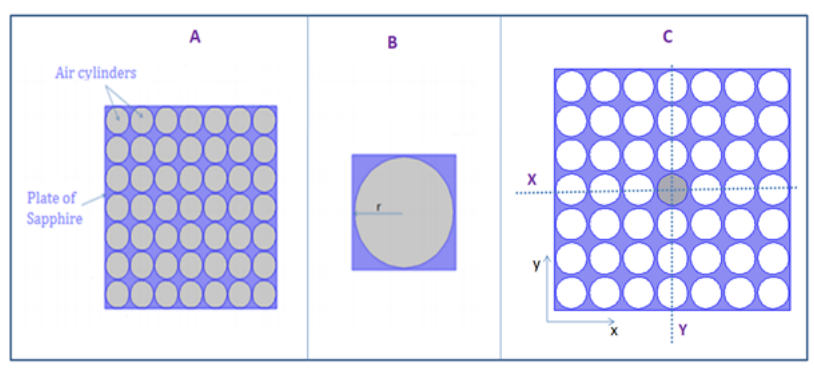

Figure 1. a) Two-dimensional photonic crystal composed of cylinders arranged periodically in a square arrangement on a plate of Sapphire. (b) Elementary cell. (c) Plans of symmetry.

\section{Dual photonic and phononic band gaps}

In this systematic study, the calculation of the band structure has been done to the main directions of the first Brillouin zone of the square lattice for Sapphire, that is to say along the closed path $\Gamma-X-M-\Gamma$, where $\Gamma X$ and $\Gamma M$ the directions of the first Brillouin zone. In the band structures presented in the paper, the frequencies are given in the dimensionless unit $\Omega=\omega a / 2 \pi c$ where $c$ is the velocity of light in vacuum for electromagnetic waves and the transverse velocity of sound in sapphire for elastic waves. Photonic simulation is done in the range of microwave frequencies where the dielectric tensor of sapphire is anisotropic and the refractive indices are high $\varepsilon_{\|}=11.5$ and $\varepsilon_{\perp}=9.3$. Obviously spoken, obtaining a photonic band gap in an infrared frequency range is more difficult because of the low refractive index of the sapphire in this range $\left(n_{\text {Sapphire }}=\right.$ 1.75). Figure 2 shows the results obtained for the TE and TM polarizations, meaning the electric field and the magnetic field are parallel to the axis of the cylinders.

The dispersion curves in Figure 2 show the existence of a common gap between the TE mode and TM mode of the Sapphire matrix for a fill factor is 0.66 . So these results are of great importance in view of completion of the optical and acoustic band gaps: this is the phoxonic crystal that crossed the world of telecommunications and radar.

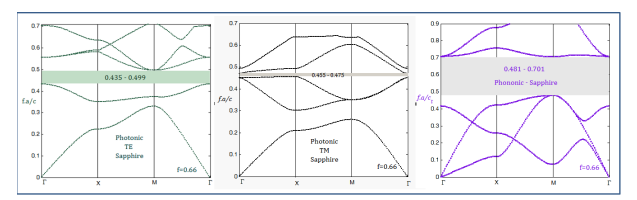

Figure 2. Band structure of a two-dimensional square lattice of air holes immersed in a matrix of Sapphire in TM and TE mode.

\subsection{Photonic and phononic structure with defect}

The objective in this section is the confinement of energy in a cavity. Punctuel defects completely surrounded by the photonic and phononic crystal, allowed to frequencies within the band gap to penetrate and confine, the photonic and phononic crystal with their defects behave like almost perfect mirrors. When we speak about optical cavities, the application that immediately comes to mind is the development of lasers. Just insert an active material within the cavity to obtain a microlaser.

The periodic structure is composed of a square array of air cylinders in a matrix of Sapphire $\left(\mathrm{Al}_{2} \mathrm{O}_{3}\right)$. The width of the band gap of the defect-free crystal is proportional to the confinement within the cavity. This condition is fulfilled when the filling fraction is large. In this calculation, the radius of the rolls of air has been chosen equal to $0.4584 a$, where $a$ is the lattice parameter, which corresponds to a filling factor of $f=\pi r^{2} / a^{2}=0.66$, the defect created in the structure is an alternative default; the air hole in the center of the super cell is replaced by a silicon hole of same radius.

In this part, we will search the default mode when we break the periodicity of the material. then we use the concept of supercell. A supercell comprises a plurality of primitive cells. In this case, there is a supercell consisted of $7 \times 7$ primitive cells, meaning the cavity is repeated along the $x$ and $y$ directions with a super-period equal to $L=7 a$, where $a$ is the lattice parameter, wherein a cavity is created by modifying a hole. 
Figure 3 shows the mapping of fields of the 3 TM modes, 2 TE optical modes and 6 phononic modes localized in their respective photonic and phononic band.

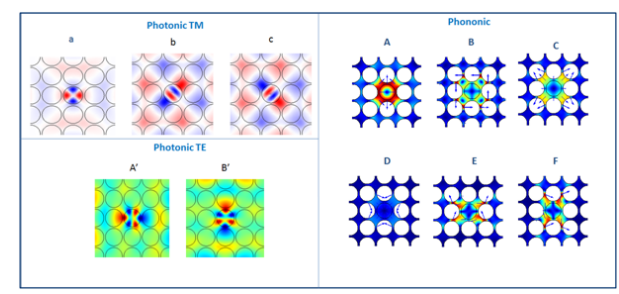

Figure 3. Mapping of the elastic and electromagnetic fields in the plan $(\mathrm{x}, \mathrm{y})$ for the photonic and phononic modes located in their respective band gap. For each mode of the cavity, the blue arrows indicate the magnitude and direction of the displacement field vector.

Photonic and phononic modes are classified according to their symmetry, or symmetrical (even (e)) antisymmetric (odd (o)), relative to the two planes of symmetry $\mathrm{X}$ and $\mathrm{Y}$ of the cavity (Figure 1.C). An analysis of the symmetry of the modes has been realized from the distributions of the fields in Fig. 3. As a result, the optical modes (b) and (c) are of symmetry (oo), (a) is of symmetry (ee), (A') and (B') are of symmetry (oe) and (eo) respectively.

The phononic modes (A) and (D) are of symmetry (ee), (B) and (C) are of symmetry (oo). The other two modes (E) and (F) are degenerate and from fields of presentations, (oe) and (eo) are the respective symmetries of (E) and (F). The description of each phonon cavity mode during a vibration period is deducted from the direction of blue arrows, animations and modes of field maps. For the mode (A), the cavity is alternately stretched along one side of the square lattice, while the other side contracts. Mode (B) is a torsional mode perpendicular to the axis of the cavity with a rotation in the counterclockwise direction in the outer part of the cavity. The evolution in time of the mode (C) is such that the cavity is stretched along a diagonal and contracted along the other for a half acoustic period. The temporal evolution of the mode (D) substantially shows a breathing motion with homothetic deformation of the shape of the cavity. Finally, the field distribution of the modes (E) and (F) is orthogonal.

Punctuel defects completely surrounded by the photonic and phononic crystal, allowed to frequencies within the band gap to penetrate and confine, the photonic and phononic crystal with their defects behave like almost perfect mirrors. When we speak about optical cavities, the application that immediately comes to mind is the development of lasers. Just insert an active material within the cavity to obtain a micro laser.

\section{Conclusion}

In this work, we have simulated the propagation of electromagnetic and elastic waves in phononic and photonic crystals, and we have demonstrated that a periodic array of air holes deposited on a sapphire matrix exhibits simultaneous phononic and photonic complete band gaps in the square lattice. The confinement of electromagnetic and elastic energy is obtained by inserting a substitution defect in a silicon slab presenting simultaneously a phononic and a photonic band gap. The design of phoxonic structures depends on the geometrical parameters crystal, which were chosen, based on the finite element analysis of a perfect phoxonic crystal of circular holes. This does not stop only at the choice of the type of network but also the choice of materials, their respective cuts, or inclusions rays.

\section{References}

1. E. Yablonovitch, J. Opt. Soc. Am. B 10, 283, (1993).

2. J. D. Joannopoulos, S. G. Johnson, J. N. Winn, and R. D. Meade, Photonic Crystals: Molding the Flow of Light Book title (Princeton University Press, 2008).

3. M. S. Kushwaha, P. Halevi, L. Dobrzynski, and B. Djafari-Rouhani, Phys. Rev. Lett. 71, 2022 (1993).

4. Y. Pennec, J. Vasseur, B. Djafari-Rouhani, L. Dobrzynski, and P. A. Deymier, Surf. Sci. Rep. 65, 229, (2010).

5. T. Gorishny, M. Maldovan, C. Ullal, and E. L. Thomas, Phys. World, 18, 24 (2005).

6. M. Eichenfield, J. Chan, R. M. Camacho, K. J. Vahala, and O. Painter, Nature (London) 462, 78 (2009).

7. M. Eichenfield, J. Chan, R. M. Camacho, K. J. Vahala, and O. Painter, Nature (London) 459, 550 (2009).

8. M. Maldovan et E.L. Thomas: Simultaneous complete elastic and electromagnetic band gaps in periodic structures. Applied Physics B, 83(4): 595- 600, (2006).

9. Martin Maldovan et Edwin L. Thomas: Simultaneous localization of photons and phonons in twodimensional periodic structures. Applied Physics Letters, 88 (25): 251907 (2006).

10. Said Sadat-Saleh, Sarah Benchabane, Fadi Issam Baida, Maria-Pilar Bernal et Vincent Laude: Tailoring simultaneous photonic and phononic band gaps. Journal of Applied Physics, 106 (7): 074912 (2009).

11. S. Sadat-Saleh, S. Benchabane, F.I. Baida, MariaPilar Bernal et S. Laude: Simultaneous photonic and phononic band gaps in a two-dimensional lithium niobate crystal. In Ultrasonics Symposium (IUS), 2009 IEEE International, pages 1118- 1121 (2009).

12. B. Djafari Rouhani, Y. Pennec, E.H. Boudouti, J.O. Vasseur, Y. Hassouani, C. Li, A. Akjouj et D. Bria: Band gap engineering in simultaneous phononic and photonic crystal slabs. Applied Physics A, 103(3): 735- 739 (2011).

13. Y. El Hassouani, C. Li, Y. Pennec, E. H. El Boudouti, H. Larabi, A. Akjouj, O. Bou Matar, V. Laude, N.Papanikolaou, A. Martinez et B. Djafari Rouhani : Dual phononic and photonic band gaps in a periodic array of pillars deposited on a thin plate. Phys. Rev. B, 82: 155405 (Oct 2010).

14. Y. Pennec, B. Djafari Rouhani, E. H. El Boudouti, C. Li, Y. El Hassouani, J. O. Vasseur, N. Papanikolaou, S. Benchabane, V. Laude, et A. Martinez: Band gaps and waveguiding in phoxonic silicon crystal slabs. CHINESE JOURNAL OF PHYSICS, 49: 100 (Feb 2011).

15. Saeed Mohammadi, Ali A. Eftekhar, Abdelkrim Khelif et Ali Adibi: Simultaneous two dimensional 
phononic and photonic band gaps in opto-mechanical crystal slabs. Opt. Express, 18(9): 9164- 9172 (Apr 2010).

16. L. Brillouin, Diffusion de la lumière et des rayons $\mathrm{x}$ par un corps transparent homogène, influence de l'agitation thermique. Ann. Phys., 17, 88-122 (1922). University Press (1995).

17. Q. Rolland, M. Oudich, S. El-Jallal, S. Dupont, Y. Pennec, J. Gazalet, J. C. Kastelik, G. Leveque, and B. Djafari-Rouhani. Appl. Phys. Lett. 101, 061109 (2012).

18. S. El-jallal, M. Oudich, Y. Pennec, B. DjafariRouhani, A. Makhoute, Q. Rolland, S. Dupont, and J. Gazalet. J. Phys.: Condens. Matter 26, 015005 (2014).

19. S. El-Jallal, M. Oudich, Y. Pennec, B. DjafariRouhani, V. Laude, J.-C. Beugnot, A. Martinez, J. M. Escalante, and A. Makhoute. Phys. Rev. B 88, 205410 (2013).

20. M. Oudich, S. El-Jallal, Y. Pennec, B. D. Rouhani, J. Gomis-Bresco, D. Navarro-Urrios, C. M. S. Torres, A. Martinez, and A. Makhoute, Phys. Rev. B 89, 245122 (2014).

21. J. Gomis-Bresco, D. Navarro-Urrios, M. Oudich, S. El-Jallal, A. Griol, D. Puerto, E. Chavez, Y. Pennec, B. D. Rouhani, F. Alzina, A. Martinez and C.M. Sotomayor Torres. Nature communications 5, 4452 (2014).

22. D. Navarro-Urrios, J. Gomis-Bresco, S. El-Jallal, M. Oudich, A. Griol, A. Pitanti, N. Capuj, A. Tredicucci, F. Alzina, A. Griol, Y. Pennec, B. Djafari-Rouhani, A. Martinez, and C. M. Sotomayor Torres. "Dynamical back-action at $5.5 \mathrm{GHz}$ in a corrugated optomechanical beam”. AIP Advances 4, 124306 (2014).

23. D. Bria, M. B. Assouar, M. Oudich, Y. Pennec, J. Vasseur, and B. Djafari-Rouhani, J. Appl. Phys. 109, 014507 (2011).

24. E. Yablonovitch, Phys. Rev. Lett. 58, 2059 (1987).

25. A. Taflove, the Finite-Difference Time-Domain Method. (Artech, Boston (1998)).

26. Yariv. A and Yeh. P Optical Waves in Crystals (New York: Wiley 1984).

27. Renosi P, Sapriel J and Djafari Rouhani B 1993 Proc. 5th Int. Conf. on Indium Phosphide and Related Material (Paris) pp 592-5 doi: 10. 1109/ICIPRM. 1993. 380551 see also Renosi P and Sapriel J 1994. Appl. Phys. Lett. 642794. 